J.R. GREER W.D. NIX

\section{Size dependence in mechanical properties of gold at the micron scale in the absence of strain gradients}

Department of Materials Science and Engineering, Stanford University, Stanford, CA 94305-2205, USA

\section{(C) Springer-Verlag 2007}

Subsequent review of three figures in this paper detected errors that should be corrected. A review of the data leading to Fig. 4 of the paper has revealed that the two points corresponding to strengths of $1 \mathrm{GPa}$ and $4 \mathrm{GPa}$ are in error and should be omitted. In our earliest tests the circular trench surrounding each pillar was not large enough to accommodate the precise positioning of the flat punch indenter tip, with the consequence that the sloped sides of the indenter came into contact with the outside edge of the trench. This causes the force to be supported by both the pillar and the edge contact and leads to computed strengths that are erroneously high. We believe that these two specific points were caused by those errors. The top two star points in Fig. 6 are the same ones and should also be omitted from that figure. We have no reason to doubt the validity of the remaining strengths, and therefore of any other data presented in these figures.

Also, the stress-strain curve shown in Fig. 2 was generated from the load-displacement data in that figure and the
Appl. Phys. A 80, 1625 (2005)

DOI 10.1007/s00339-005-3204-6

Published online: 30 March 2005

shown pillar dimensions. Subsequent analysis of the data indicated that the pillar diameter was slightly larger than that shown $(400 \mathrm{~nm}$ instead of $350 \mathrm{~nm}$ measured in the top $1 / 3$ of the pillar). Since the bottom of the pillar was obscured by the trench surrounding the pillar, we subsequently used the modulus to obtain a better measure of the length of the pillar. That procedure led to a height of $1960 \mathrm{~nm}$ instead of $1600 \mathrm{~nm}$ shown in the published paper. The stress-strain curve corresponding to this revised geometry is shown below and should be used in place of Fig. $2 b$.

Similar corrections have been published for our similar, but more detailed, paper in Acta Materialia: J.R. Greer, W.C. Oliver and W.D. Nix, Corrigendum to 'Size dependence in mechanical properties of gold at the micron scale in the absence of strain gradients', Acta Mater. 53(6), 1821 (2005).

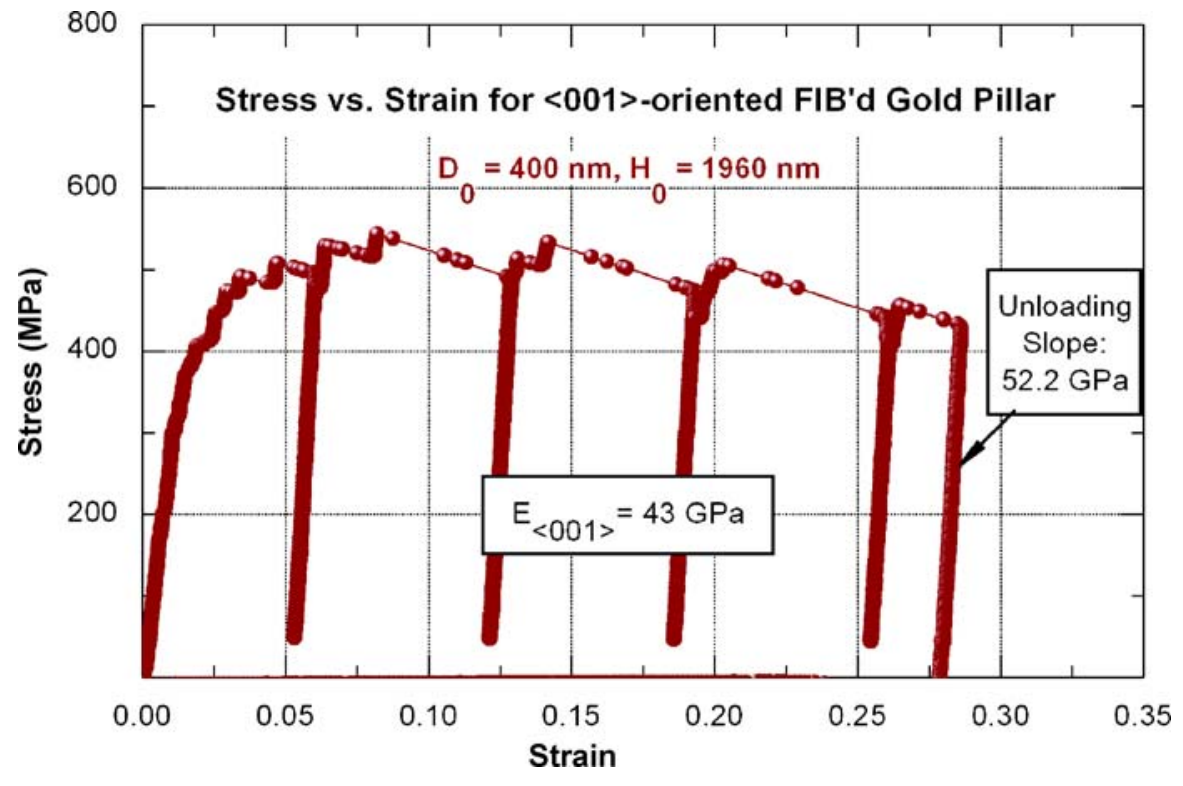

Fax: +1-650-725-4034, E-mail: nix@stanford.edu The online version of the original article can be found at http://dx.doi.org/10.1007/s00339-005-3204-6 